Mots. Les langages du politique

\title{
Les mots de l'écologie, 25 ans après. Circulation des discours et des notions
}

The words of ecology, 25 years later. The circulation of discourses and of notions Las palabras de la ecología, 25 años despues. Circulación de los discursos y de las nociones

Valérie Bonnet et Albane Geslin

\section{(2) OpenEdition}

\section{Journals}

Édition électronique

URL : https://journals.openedition.org/mots/24186

DOI : $10.4000 /$ mots. 24186

ISSN : 1960-6001

Éditeur

ENS Éditions

\section{Édition imprimée}

Date de publication : 5 mars 2019

Pagination : 9-14

ISBN : 979-10-362-0130-1

ISSN : 0243-6450

\section{Référence électronique}

Valérie Bonnet et Albane Geslin, «Les mots de l'écologie, 25 ans après. Circulation des discours et des notions », Mots. Les langages du politique [En ligne], 119 | 2019, mis en ligne le 01 janvier 2021, consulté le 24 avril 2022. URL : http://journals.openedition.org/mots/24186 ; DOI : https://doi.org/10.4000/ mots. 24186 


\section{Les mots de l'écologie, 25 ans après. Circulation des discours et des notions}

25 ans après la parution d'un numéro consacré à l'écologie politique, la revue Mots. Les langages du politique remet sur le métier la question des mots et des discours de l'écologie, reprenant, pour ce numéro, le titre d'un article alors écrit par Geneviève Petiot. Où en sont aujourd'hui les discours sur l'environnement? L'écologie est installée dans le paysage politique : au-delà des partis dits écologistes, les candidats à la présidentielle française présentent tous un volet - plus ou moins - environnemental dans leur programme. Les dernières élections en Bavière qui ont vu la montée en puissance des Grünen, les manifestations demandant la prise en compte des perspectives environnementales à la suite de la démission de Nicolas Hulot, la mise à l'agenda politique et médiatique des « $\mathrm{COP}^{1}$ » sur le changement climatique ou la publication du dernier rapport du GIEC2 indiquent l'inscription durable et forte de ces discours dans l'espace public. La création même du GIEC est de fait un signe de la prise en compte des problématiques du réchauffement climatique par les instances internationales (l'attribution du prix Nobel de la paix à ce rassemblement de chercheurs en climatologie établit l'installation des réflexions environnementales sur le plan mondial, global, et non pas seulement international). Au même titre, la presse institutionnelle s'est enrichie de rubriques consacrant à l'environnement des espaces dédiés, et plus largement, ce thème est diversement abordé dans les médias, y compris dans les pages «Politique internationale».

Depuis 1994, les revues de sciences de la vie et de sciences humaines et sociales ont considérablement accru leur corpus en la matière, accueillant de manière récurrente des articles portant sur cet objet, et l'on dénombre une production notable de thèses traitant de diverses controverses environnementales. Le grand nombre de propositions reçues en réponse à l'appel à

1. Conférence des parties à la Convention-cadre des Nations unies sur les changements climatiques.

2. Groupe d'experts intergouvernemental sur l'évolution du climat.

Université Toulouse 3-Paul Sabatier, LERASS

valerie.bonnet@uni-tlse3.fr

Sciences Po Aix, DICE-CERIC

albane.geslin@sciencespo-aix.fr 
contributions qui a nourri ce numéro a été un indicateur de vitalité du sujet, tout comme de sa diversité :

- Diversité des méthodes3 et des disciplines impliquées (sociologie, anthropologie, science politique, sciences du langage et du discours, sciences de l'information et de la communication, droit, etc.), des observables langagiers (formules, slogans, arguments) et des degrés de construction idéologique ;

- Diversité des terrains et sujets (types de nuisances environnementales, controverses autour des énergies, débats sur le réchauffement climatique, problématique des «réfugiés» environnementaux, modification des écosystèmes, enjeux de santé publique), et de l'empan des problèmes soulevés (avenir de populations, avenir des écosystèmes, enjeux locaux ou globaux);

- Diversité des acteurs (institutions nationales, supranationales, internationales, syndicats, partis, associations, élus locaux ou européens, citoyens) et des producteurs de discours (politiques, scientifiques, journalistes, citoyens, militants, photographes-vidéastes), diversité qui induit une diversité des genres de discours portant sur l'environnement (rapports, textes de loi, conventions, professions de foi, discours politiques, articles de presse, documentaires, billets, décisions de justice, tweets, etc.), et des lieux de production et de circulation de ces discours (institutions, manifestations, réseaux sociaux, médias, etc.).

La circulation des matériaux langagiers et des discours, entre acceptabilité, réappropriation et détournement, est certainement l'un des points majeurs traversant les propositions et les articles. Le trajet des notions, des concepts, des propos peut être appréhendé sur le plan des relations intergroupes, celles des acteurs des crises et controverses environnementales; il en est de même de la reprise des termes technoscientifiques, y compris dans une perspective de banalisation. C'est en effet dans le lexique que se joue sans doute avec le plus d'acuité la rencontre entre le scientifique et le social (voir par exemple Gaudin, 1993), l'expert et la société civile, le militant et l'opérateur économique, si l'on songe aux problématiques d'acceptabilité des termes, des querelles de nomination. Ainsi, la notion de formule développée entre autres par Alice Krieg-Planque rend compte d'expressions dont le dessein est de contraindre le débat public, neutraliser les éventuelles conflictualités et stabiliser les polémicités (voir dans le contexte précis de ce volume Krieg-Planque, 2010). Pour citer Lamria Chetouani et Maurice Tournier (1994, p.4), rien n'est plus politique que l'acte de désigner, et, de fait, les logiques de nomination et de catégorisation inhérentes (l'accident de Three Miles Island versus la catastrophe de Tcherno-

3. Le travail quantitatif souvent évoqué et employé signifie la présence de très gros corpus, indice de la mobilisation de l'espace public autour de ces problématiques. 
byl), les choix lexicaux, peuvent être vecteurs d'axiologie, convoquer des discours autres, par le truchement de divers tropes sémantiques (antonomase, synecdoque, métonymie, métaphore), de préfixes (alter-, éco-, etc.) ou de néologismes (on pense à écocide ou anthropocène par exemple).

Le second type de circulation des discours, des notions et des concepts peut être appréhendé selon l'axe du temps. Les travaux montrent l'importance des temporalités dans ce domaine, et notamment des rappels, des dimensions mémorielles qu'il convient de prendre en compte ou d'écarter, en fonction des effets visés. Les crises environnementales (les pluies acides, Fukushima, Tchernobyl, l'érosion de la diversité biologique) jouent un rôle primordial dans le déplacement de l'écologie de la sphère scientifique et technique vers le champ du politique. Tout comme les appels, grands sommets et conférences (Rio, Kyoto, La Haye, Copenhague, Grenelle, Paris, etc.), ces grandes crises constituent des événements, provoquant des moments discursifs (Moirand, 2007), leurs dénominations circulant dans l'interdiscours. Le caractère disruptif (Fukushima), initial (accident de Three Miles Island, la conférence de Rio) de ces crises, mais aussi leur statut de prototype ou de parangon, qui les amène à dépasser leur catégorie de référence (on songe à des expressions comme Tchernobyl aviaire, Tchernobyl chimique), explique leur forte présence mémorielle ou encore leur rémanence. Enfin, la dimension temporelle est également prégnante dans la représentation de l'avenir, ou encore dans la proximité grandissante des échéances exigeant la prise en charge des problèmes publics (symptomatique à cet égard est le développement récent des contestations judiciaires engagées par des associations ou groupement de personnes contre les autorités publiques - Pays-Bas, France, Union européenne, ÉtatsUnis notamment - mettant en cause leur manque de réactivité et d'ambition face à l'urgence de la lutte contre les changements climatiques).

À ce courant nourri par la crainte de la dangerosité d'une science mal maitrisée, il convient d'adjoindre le mouvement dit décroissant, qui prendrait ses sources dans une opposition à la société industrielle et capitaliste. La technoscience semble donc constituer le pivot articulant ces deux tendances de la pensée écologiste, ces deux univers idéologiques.

Le périmètre de la question écologique parcourt les travaux. Qu'associe-ton à celle-ci (l'aire sémantique du terme est en effet en expansion) ? Que projette-t-on dans l'écologie (une pensée envisageant un développement durable ou dysphorique car catastrophiste) ? Et surtout quelle est la portée de la problématique environnementale (le terme environnement lui-même étant particulièrement polysémique4) : le social, l’humanité, le vivant, la planète? Les

4. Voir à ce propos les travaux de France Guérin-Pace et Philippe Colomb (1998), de Jacqueline Picoche (2003), d'Olivier Godard (2015). Ces textes, qui abordent le terme environnement selon des méthodologies différentes (lexicologie, statistique textuelle, analyse conceptuelle) en raison des disciplines d'appartenance de leurs auteurs (sciences du langage, démographie, 
moteurs idéologiques, d'action et de motivation sont variables, ainsi que le montre le présent dossier.

Celui-ci propose six articles dont l'empan géographique et spatial soumet à différentes focales les problématiques environnementales abordées dans leurs dimensions politiques et sociales.

En ouverture, la contribution de Béatrice Fracchiolla aborde la construction et les évolutions du discours écologiste des Verts français et italiens des années 2000. Adoptant la perspective d'une analyse sémantique et historique, fondée sur des entretiens auprès d'adhérents militants Verts et Verdi, l'autrice met en évidence la dialectique du proche et du lointain commune aux deux courants, soulignant les liens existants entre l'écologie comme science, comme concept et comme idéologie. L'ensemble des entretiens permet également de rendre compte de la place et de la polysémie des termes environnement et écologie non seulement dans chacun des discours militants, mais aussi dans les statuts des partis politiques.

Kjersti Fløttum, Øyvind Gjerstad et Anje Müller Gjesdal explorent la représentation de l'avenir telle qu'elle se présente dans les blogs francophones portant sur les changements climatiques. Analysé dans une perspective lexicosémantique, le corpus portant sur les périodes 2009-2010 et 2013-2014 montre une dominance des perspectives dysphoriques. Mais c'est surtout en contraste avec les blogs anglophones que ce résultat est éclairant. En effet, ceux-ci affichent un discours qui dévoilerait davantage de représentations positives de l'avenir, construites autour de la notion de sustainability. La mise en perspective de ce travail à la lumière de la COP 24 est particulièrement pertinente dans une réflexion questionnant la complexité du discours environnemental, et les moyens de rendre le discours environnemental engageant.

Dans un contexte plus spécifique, l'étude de Yeny Serrano, Christine Heimlich, Cyrille Bodin, Philippe Chavot, Anne Masseran et Jean Zoungrana porte quant à elle, non pas sur les représentations, mais sur l'usage d'une expression - la géothermie profonde n'est pas mature - dans le cadre de quatre projets soumis à enquêtes publiques réalisées dans l'Eurométropole de Strasbourg. Empruntée à un scientifique, cette expression a irrigué tant les rapports officiels des enquêteurs publics que le discours des parties prenantes, qu'il s'agisse des opérateurs responsables des projets ou des opposants (citoyens, associations ou élus). L'expression, devenant une formule-argument circulant dans les discours des acteurs, a subi un réagencement sémantique du fait de son appropriation par les opposants à la technologie en cause.

Dans la même perspective, Marieke Stein propose une analyse des polémicités accompagnant la désignation du Coal Bed Methane. Lieu d'enjeux argumen-

méta-économie), indiquent que cette question pointe de manière récurrente dans le champ des sciences humaines et sociales. 
tatifs et partisans, le terme n'a toujours pas trouvé de traduction française. Par une analyse des leviers rhétoriques et lexicaux, l'autrice met au jour les structurations du débat, soulignant les diverses logiques et stratégies (clarification, acceptabilité, neutralisation) des acteurs (opposants, Commission d'enrichissement de la langue française, experts), les nominations proposées visant à infléchir les représentations. Si la littérature a montré qu'en raison de divers enjeux politiques ou industriels, les technosciences ont souvent été le lieu de la régulation et de la négociation lexicale (Delavigne, 2006 ; Bonnet, 2008), l’originalité de ce travail est de croiser réflexion sur les controverses et réflexion sur la planification de corpus.

Tout comme M. Stein, Laurence Vignes souligne que le syntagme à visée scientifique des promoteurs, "gaz non conventionnel», neutralisant, est rejeté par ses contempteurs pour plébisciter la nomination « gaz de schiste». Par son analyse diachronique des inscriptions sur banderoles et calicots contre le gaz de schiste (de 2011 à 2016), cette autrice montre les divers usages de l'interdiscours dans les jeux de mots et autres slogans polyphoniques. Est construit un thésaurus, qui renvoie aux combats historiques tant écologiques, identitaires, qu'antitotalitaires, comme au contexte politique immédiat. Est ainsi modifiée la portée d'une lutte environnementale, qui devient également une lutte pour les valeurs démocratiques.

La notion d'interdiscours est également convoquée par le travail de Guillaume Carbou, qui interroge les représentations de la technologie. Cet auteur appréhende en effet les discours écologistes sous l'angle de la protestation romantique, sur la base de deux corpus : d'une part six auteurs considérés comme des fondateurs ou inspirateurs de l'écologie politique (Bernard Charbonneau, André Gorz, Ivan Illich, Henry David Thoreau, Arne Naess et Murray Bookchin) et d'autre part des contestations d'internautes contre de grands projets d'aménagement du territoire sur des sites de presse en ligne. Le romantisme y est perçu comme une réaction au désenchantement du monde, comme une protestation contre les diverses aliénations de l’individu (économico-politique, philosophique, épistémique et technique), mais également comme un «réoutillage» de la société. L'analyse des discours sous l'angle de l'écologie romantique permet à l'auteur d'expliquer pourquoi certaines protestations s'élèvent contre des projets a priori écologiques et pourquoi des causes locales attirent des opposants venant de zones éloignées. Il note enfin que, dans une perspective romantique, à la différence de l'environnementalisme, la nature n'est qu'un prétexte ou un symbole. 


\section{Références}

BONNET Valérie, 2008, "Les enjeux socio-politiques de la nomenclature chimique de 1787 ", dans Langue et politique en France à l'époque des Lumières, S. Große et C. Neis éd., Francfort-sur-le-Main, Domus Editoria Europaea, p.63-82.

Chetouani Lamria, Tournier Maurice éd., 1994, Environnement, Écologie, Verts [numéro thématique], Mots. Les langages du politique, nº 39.

GuÉrin-PACe France, Collomb Philippe, 1998, « Les contours du mot “environnement” : enseignements de la statistique textuelle », L'Espace géographique, t.XXVII, n ${ }^{\circ} 1$, p. 41-52.

DELAVIGNE Valérie, 2006, «La formation du vocabulaire de la physique nucléaire : quelques jalons », dans Aspects diachroniques du vocabulaire, F. Gaudin et D. Candel éd., Mont-Saint-Aignan, Publications des universités de Rouen et du Havre, p. 89-107.

KRIEG-PLANQUE Alice, 2010, «La formule "développement durable” : un opérateur de neutralisation de la conflictualité », Langage et société, n0 134, p. 5-29.

GAUDIN François, 1993, Pour une socioterminologie : des problèmes sémantiques aux pratiques institutionnelles, Mont-Saint-Aignan, Publications de l'université de Rouen.

GodARD Olivier, 2013, Environnement et développement durable : une approche métaéconomique, Louvain-la-Neuve, De Boeck.

MoIRAND Sophie, 2007, «Discours, mémoires et contextes : à propos du fonctionnement de l'allusion dans la presse », Corela, hors-série n 6 , https://journals.openedition. org/corela/1567 (consulté le 7 mars 2017).

Petıot Geneviève, 1994, "Les mots de l'écologie », Mots. Les langages du politique, n०39, p. 69-78.

PICOCHE Jacqueline, 2003, «Champ actanciel du mot environnement et discours sur l'environnement», dans L. Panier et S. Rémi-Giraud éd., La polysémie ou l'empire du sens : lexique, discours, représentations, Lyon, Presses universitaires de Lyon, p. 255-262. 